\title{
Effects of miRNA-130a on the proliferation and apoptosis of glioma cell lines
}

\author{
KE LU ${ }^{1}$, HECHUN SHEN ${ }^{1}$, SHENG ZHU $^{1}$, SHUIQING BI ${ }^{2}$ and SHENGTIAN WU ${ }^{1}$ \\ ${ }^{1}$ Department of Neurosurgery, The Second Nanning People's Hospital, Nanning, Guangxi 530031; \\ ${ }^{2}$ Department of Neurosurgery, The First Affiliated Hospital of Guangxi Medical University, \\ Nanning, Guangxi 530021, P.R. China
}

Received December 8, 2017; Accepted May 24, 2018

DOI: $10.3892 / 01.2018 .8878$

\begin{abstract}
Regulatory ability of micro-ribose nucleic acid-130a (miRNA-130a) in the proliferation and invasive growth of human brain glioma cells and its mechanism were investigated. RT-qPCR was used to analyze expression of miRNA-130a in U-87MG glioma specimens; lipidosome was used to mediate miRNA-130a mimic transfecting glioma cells and the expression of miRNA-130a was detected by using RT-qPCR after transfection; methyl thiazolyl tetrazolium (MTT) assay and flow cytometry (FCM) were adopted to evaluate the changes in biological characteristics of cell growth and proliferation; the migration and invasion abilities of tumor cells were measured through scratch assay and Transwell in vitro cell migration assay. In miRNA-130a mimic-transfected U-87MG cells, RT-qPCR showed that the expression of miRNA-130a was upregulated; MTT assay and FCM revealed that the cell growth was strengthened; scratch assay and Transwell in vitro cell migration assay verified that the migration and invasion abilities of cells were enhanced. In conclusion, the high expression of miRNA-130a can promote growth and invasion, indicating that miRNA-130a can be considered as a candidate target of gene therapy for glioma.
\end{abstract}

\section{Introduction}

According to an epidemiological survey, the incidence rate of neuroglioma has been significantly increased over the past 20 years. Neuroglioma, abbreviated as malignant brain tumors, accounts for $40 \%$ or even higher of all primary brain tumors (1). The death ranking announced by the World Health Organization (WHO) shows that glioma ranks second among death causes of tumor patients aged below 34 years and is

Correspondence to: Dr Ke Lu, Department of Neurosurgery, The Second Nanning People's Hospital, 13 Dancun Road, Jiangnan, Nanning, Guangxi 530031, P.R. China

E-mail:khcn75@163.com

Key words: miRNA-130, glioma, cell line, proliferation and apoptosis, transfection ranked third among those of middle-aged and elderly tumor patients (2). Although the treatment of glioma patients has obtained a certain effect due to the remarkable advances in operation and other treatment methods, the average survival time is only 9-12 months with poor prognoses. Moreover, the main difficulties for treating glioma are metastasis and relapse (3).

Micro-ribose nucleic acid (miRNA) is a kind of non-coding single-stranded RNA with the length of $~ 19-22$ nucleotides (nt) and a main active regulatory factor in biological bodies at present. miRNA itself does not have the function of translating proteins, but can adjust the expression of target genes through the transcription by the complementary pairing with 3 ' untranslated region (UTR) base sequences (4). In the adjusting process, miRNA leads to differential expression of genes, causing degradation and translation inhibition of messenger RNAs (mRNAs) of migrating and invading target genes concerned (5). Literature (6) has reported that miRNA-130a is relatively highly expressed in glioma tissues, but few studies exist on the function of glioma. This study investigated the effects of miRNA-130a on the proliferation, migration and invasion of glioma cell lines through in vitro experiments.

\section{Materials and methods}

Main experimental materials. Glioma cell lines (U-87MG) and human astrocytes (HA) 1800 were purchased from the Cell Bank of Shanghai Institute of Biochemistry and Biology, CAS. The U-87MG is different from that of the original cells but the origin is unknown (7). Fetal bovine serum (FBS) was obtained from Gibco (Thermo Fisher Scientific, Inc., Waltham, MA, USA) and high pure miRNA isolation kit was purchased from Roche (Madison, WI, USA). Both methyl thiazolyl tetrazolium (MTT) and dimethyl sulphoxide (DMSO) were obtained from Sigma-Aldrich (St. Louis, MO, USA) and Annexin V-fluorescein isothiocyanate (FITC) was purchased from Bestbio (Shanghai, China, http://bestbio.bioon.com.cn/). Lipofectamine ${ }^{\mathrm{TM}} 2000$ transfection reagent was purchased from Invitrogen (Thermo Fisher Scientific, Inc.). miRNA-scramble, miRNA-130a-mimics and miRNA-130a-inhibitor sequences were synthesized by GenePharma Co., Ltd. (Shanghai, China) (Table I).

The study was approved by the Ethics Committee of The Second Nanning People's Hospital. (Nanning, China). 
Table I. Primer sequences used in this study.
Items
Primer sequences

miRNA-130a-inhibitor 5'AUGCCCUUUUAACAUUGCACUG-3'

miRNA-130a-mimics 5'-AUGCCCUUUUAACAUUGCACUG-3'

miRNA-130a-scramble 5'-CAGUACUUUUGUGUAGUACAA-3'

Cell culture. Dulbecco's modified Eagle's medium (DMEM) with $10 \%$ FBS and $1 \%$ penicillin streptomycin was used to cultivate U-87MG cells, which, were then transferred into the thermostatic incubator with $5 \% \mathrm{CO}_{2}$ at $37^{\circ} \mathrm{C}$ for cultivation, digestion and passage. Trypsin was used to count cells. The density of cells were adjusted, and they were successively inoculated into 6 -well plates as per $2 \times 10^{5}$ cells/well. Afterwards, they were cultivated in DMEM (containing $10 \%$ FBS without $1 \%$ penicillin streptomycin) for $24 \mathrm{~h}$. When the total cells were fused to $80 \%$, Lipofectamine ${ }^{\mathrm{TM}} 2000 \mathrm{kit}$ was used for transfection. In this experiment, four groups were set up: the bare group, the miRNA-130a-mimics group, the miRNA-130a-inhibitor group and the miRNA-scramble group. After $6 \mathrm{~h}$ of transfection, cells continued to be cultivated for $24 \mathrm{~h}$.

Expression of miRNA-130a in cells detected via RT-qPCR. After transfection, the total RNA of cells were extracted strictly in accordance with the instructions of high pure miRNA isolation kit, and the concentration of the extracted RNA was measured by using an ultraviolet spectrophotometer (Hitachi, Tokyo, Japan). The extracted RNA was reversely transcribed into complementary deoxyribose nucleic acid (cDNA). Afterwards, RT-qPCR was conducted for amplification detection under the following conditions: pre-degeneration at $95^{\circ} \mathrm{C}$ for $10 \mathrm{~min}$, at $95^{\circ} \mathrm{C}$ for $15 \mathrm{sec}$, at $65^{\circ} \mathrm{C}$ for $30 \mathrm{sec}$ and at $72^{\circ} \mathrm{C}$ for $30 \mathrm{sec}$, respectively; 40 cycles in total. U6 was taken as a reference gene. The expression quantity was calculated by $2^{-\Delta \Delta \mathrm{Cq}}(6)$.

Proliferation ability of cells measured via MTT assay. After $24 \mathrm{~h}$ cultivation, cells were added with $40 \mu 12 \mathrm{mg} / \mathrm{ml}$ MTT solution and continued to be cultivated for $4 \mathrm{~h}$; the culture solution in wells was sucked by using a pipette, and each well was added with $100 \mu \mathrm{l}$ DMSO. Then, they were transferred into microporous plate oscillators to vibrate for $10 \mathrm{~min}$, and the crystals in wells were dissolved. Finally, the microplate reader (Bio-Rad, Hercules, CA, USA) was used to determine the calculation results.

Invasion ability of cell determined via Transwell assay. The culture solution after $24 \mathrm{~h}$ transfection was replaced of DMEM without $10 \%$ FBS for 24 h cultivation. Trypsin ( $0.25 \%$ ) was used to digest cells, and DMEM without $10 \%$ FBS was made into cell suspension $\left(2 \times 10^{5}\right.$ cells $\left./ \mathrm{ml}\right)$. The upper chamber was added with the same cell suspension $(200 \mu \mathrm{l})$, while the lower chamber was added with $500 \mu$ l DMEM (with 10\% FBS). Three groups of the same wells were set up, additionally. The prepared cells were put into the thermostatic incubator with $5 \% \mathrm{CO}_{2}$ at $37^{\circ} \mathrm{C}$ for cultivation $(24 \mathrm{~h})$, and $24 \mathrm{~h}$ later, Transwell chambers were taken out. Sterile cotton swabs were used to wipe the Matrigel and cells in the upper chamber, and then the hematoxylin-eosin
(HE) staining was conducted. Then, 5 fields were randomly selected to count cells under a low-power microscope (BX-42; Olympus,Tokyo, Japan) 3 times.

Migration ability of cells measured via scratch assay. The transfected cells in all groups were inoculated into 6-well plates for 3 sets of the same wells, in total. When $290 \%$ cells were fused, the scratching was conducted according to the prepared transverse lines in advance, with $20 \mu$ l pipette tip perpendicular to the 6-well culture plate. Then, phosphate-buffered saline (PBS) was used to wash cells 3 times, and the cells were continued to be cultivated in DMEM containing $1 \%$ FBS. The above operations were repeated 3 times.

Detection of cell apoptosis. After cells were transfected for 48 h, 4 groups of cells were separately transferred into cone-shaped tubes which were placed on ice. Cells in plates were rinsed by $2 \mathrm{ml}$ PBS, and then, with PBS removed, tubes were added with $0.5 \mathrm{ml} 0.25 \%$ tyrisin without ethylenediamine tetraacetic acid (EDTA) for incubation. Under a microscope (BX-42; Olympus) it was observed that cells started to fall off. Afterwards, cells fully fell off the culture plates by patting, and the resulting cells were re-suspended in $1 \mathrm{xPBS}$ (with the density of $1 \times 10^{6}$ cells $/ \mathrm{ml}$ ). They were put into clean centrifugal tubes, and then Annexin V-FITC, apoptosis test fluid was added. After that, they were kept in the dark at room temperature for $5 \mathrm{~min}$. The supernatant was centrifuged at 1,200 $\mathrm{x}$ g for $5 \mathrm{~min}$, and 1XPBS was added to re-suspend cells again. Then, $10 \mu \mathrm{l}$ propidium iodide (PI) was added, and flow cytometer was used for analysis.

Statistical analysis. Statistical Product and Service Solutions (SPSS) 20.0 software package (IBM Corp., Armonk, NY, USA) was used for the statistical analysis of the data obtained through the experiment. The data were compared by using t-test between two groups, and the differences in data among many groups were analyzed with the one-way analysis of variance followed by a post hoc test (Least Significant Difference). $\mathrm{P}<0.05$ was considered to indicate a statistically significant difference.

\section{Results}

The expression level of miRNA-130a detected by RT-qPCR. After U-87MG cells were transfected separately as the bare group, the miRNA-130a-inhibitor, the miRNA-130a-mimics and the miRNA-scramble groups for $24 \mathrm{~h}$, RT-qPCR detection results showed that the expression level of miRNA-130a in the miRNA-130a-mimics group was significantly increased, compared with those in the other groups; compared with the bare and miRNA-scramble groups, differences were statistically significant $(\mathrm{P}<0.05)$; the expression level of miRNA-130a in the miRNA-130a-inhibitor group was obviously downregulated, and compared with the bare and miRNA-scramble groups, differences were also statistically significant $(\mathrm{P}<0.05)$; the difference between the bare and miRNA-scramble groups was not statistically significant (Fig. 1).

U-87MG cell proliferation activity tested via MTT assay. MTT assay results on cell proliferation activity showed that from the 3rd day, the survival rate of U-87MG cells in 


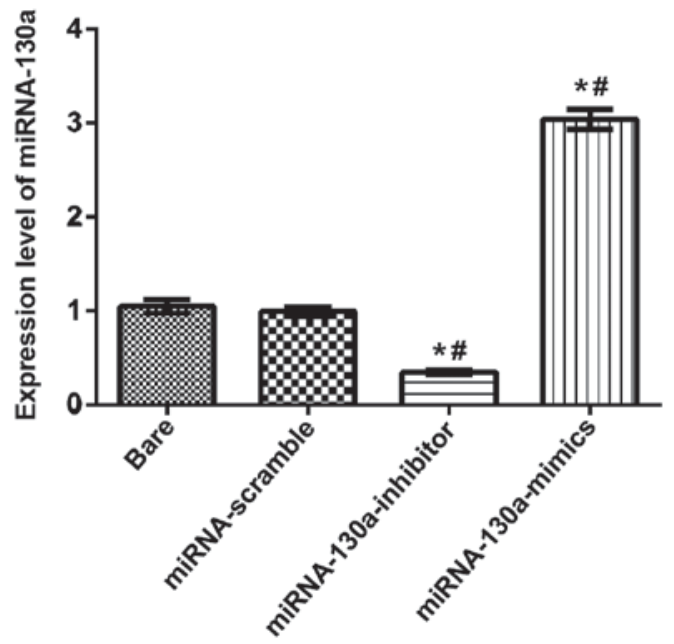

Figure 1. RT-qPCR detection. Among the bare, the miRNA-130a-inhibitor, the miRNA-130a-mimics and the miRNA-scramble groups, the comparisons of the miRNA-130a-inhibitor group with the other 3 groups are obviously statistically significant $(\mathrm{P}<0.05)$; the comparison of the miRNA-130amimics group with the bare and miRNA-scramble groups are statistically significant $(\mathrm{P}<0.05)$; the comparison between the bare and miRNA-scramble groups is not statistically significant $(\mathrm{P}>0.05)$. $\mathrm{n}=3$. ${ }^{*} \mathrm{P}<0.05$, compared with the bare group. ${ }^{*} \mathrm{P}<0.05$, compared with miRNA-scramble group. miRNA130a, micro-ribose nucleic acid-130a.

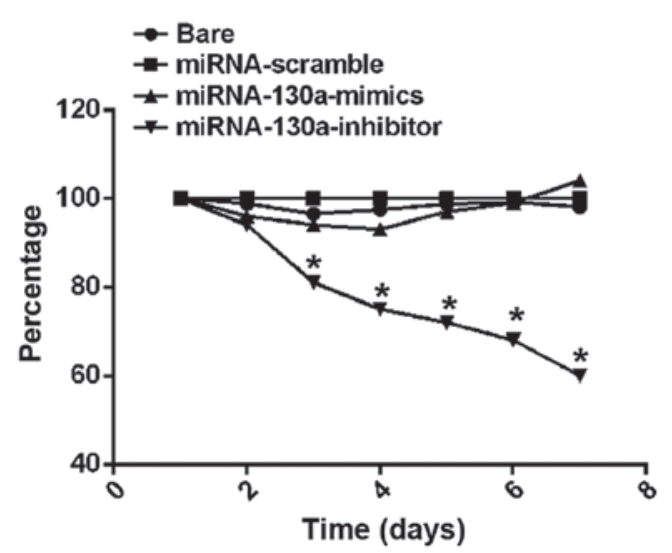

Figure 2. U-87MG cell proliferation activity measured via MTT assay. The survival rate of cells in the miRNA-130a-inhibitor group is significantly decreased compared with the bare, miRNA-scramble and miRNA-130amimics groups (" $\mathrm{P}<0.01)$. MTT, methyl thiazolyl tetrazolium; miRNA-130a, micro-ribose nucleic acid-130a.

the miRNA-130a-inhibitor group began to be significantly decreased compared with those in the bare, miRNA-scramble and miRNA-130a-mimics groups, and differences between the groups were statistically significant $(\mathrm{P}<0.01)$; on the 6th day, the cell survival rate in the miRNA-130a-inhibitor group was the lowest, and the difference from that in the bare group was statistically significant $(\mathrm{P}<0.05)$ (Fig. 2).

Expression of miRNA-130a and invasion abilities of U-87MG cells. Transwell chambers were used to test the invading ability of the miRNA-130a cells, and the results showed that the number of cells passing through the membrane in the bare, miRNA-scramble, miRNA-130a-inhibitor and miRNA-130a-mimics groups were 187.01 $\pm 8.78,154.90 \pm 8.57$, $84.71 \pm 4.41$ and $285.33 \pm 14.35$, respectively. The difference

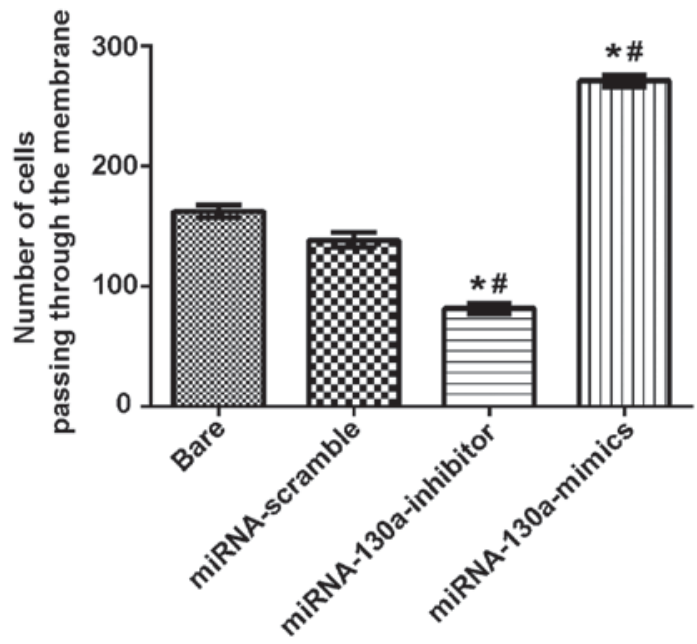

Figure 3. Expression of miRNA-130a and invasion abilities of U-87MG cells. Compared with the miRNA-scramble and bare groups, the cell invasion ability in the miRNA-130a-inhibitor group is obviously decreased with substantially statistical differences $(\mathrm{P}<0.05)$, but the invasion ability in the miRNA-130a-mimics group is significantly increased with statistically significant differences $(\mathrm{P}<0.05)$. ${ }^{*} \mathrm{P}<0.05$, compared with the bare group. ${ }^{\#} \mathrm{P}<0.05$, compared with the miRNA-scramble group. miRNA-130a, microribose nucleic acid-130a.

between the miRNA-scramble and bare groups was not statistically significant; compared with the miRNA-scramble and bare groups, the miRNA-130a-inhibitor group showed a significantly decreased invasion ability with obviously statistical differences $(\mathrm{P}<0.05)$, while the miRNA-130a-mimics group had a remarkably increased invasion ability with statistically significant differences $(\mathrm{P}<0.05)$ (Fig. 3).

Expression of miRNA-130a and migration abilities of $U$-87MG cells. Under an inverted optical microscope (BX-42; Olympus) the width of scratching wound was observed, and the results revealed that the cell migration distance in the miRNA-130a-mimics group was significantly longer than that in the miRNA-scramble and bare groups with statistically significant differences $(\mathrm{P}<0.05)$; in the miRNA-130a-inhibitor group, the migration distance was increasingly and significantly shorter than that of the miRNA-scramble and bare groups with obviously statistical differences $(\mathrm{P}<0.05)$. There were no statistically significant differences between the bare and miRNA-scramble groups (Fig. 4).

Apoptosis of glioma cells after transfection of miRNA-130a. The apoptosis of U-87MG cells varied. After only miRNA-130a was transfected, the number of apoptotic cells increased (with the apoptotic cell proportion of 3.56\%). Apoptotic cell proportions in the bare, miRNA-scramble and miRNA-130a-mimics groups were $1.62,1.81$ and $2.01 \%$, respectively after transfection, while the apoptosis in the miRNA-130a-inhibitor group (with apoptotic cell proportion of 13.54\%) showed statistical differences from those in the former three groups $(\mathrm{P}<0.01)$.

\section{Discussion}

Worldwide tumors jeopardize human health, and the occurrence and development mechanisms are complicated. 


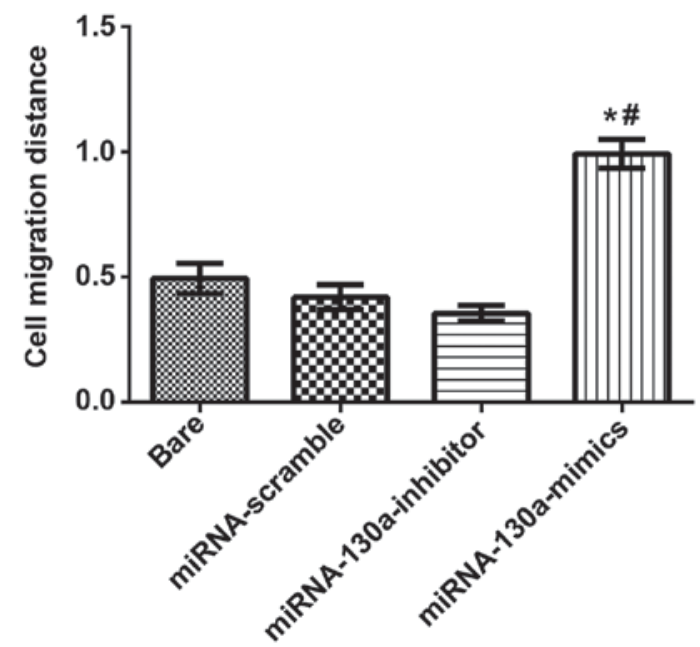

Figure 4. Expression of miRNA-130a and migration abilities of U-87MG cells. The migrating distance of the miRNA-130a-mimic group is longer than those of the miRNA-scramble group and the bare group with statistically significant differences $(\mathrm{P}<0.05)$. The difference between the bare group and the miRNA-scramble group is not statistically significant. ${ }^{*} \mathrm{P}<0.05$, compared with bare group. ${ }^{\text {"P}} \mathrm{P}<0.05$, compared with miRNA-scramble group. miRNA-130a, micro-ribose nucleic acid-130a.

According to the statistical data, the incidence rate of tumors in adults in 2013 is 3.19/100,000 in America, and the number of male patients is larger than that of female patients. The occurrence of a tumor shows a positive correlation with age. Elderly people aged $70-80$ years are vulnerable to tumors $(8,9)$. The prognosis is relatively poor, and there are many risk factors, such as age, place and size of tumor (10). Currently, the main treatment method is the combination of operation and chemoradiotherapy, and the 2-year survival rates of patients treated are remarkably increased from 10.4-26.5\% (9). However, the postoperative relapse and metastasis are prone to causing death of patients. Therefore, it is expected that an effective gene marker will be found and used to diagnose tumor patients at an early stage.

In the last 10 years, miRNA, as a new marker, has gradually attracted people's attention and is abnormally expressed in multiple tumors. Furthermore, it is involved in the occurrence and development of tumors, such as proliferation, migration and invasion and biological functions of many tumors (11-13). The differential expression of miRNA-130a occurs in many tumors, for example, its expression is downregulated in leukemia (14) patients. In cell culture in vitro, miRNA-130a can adjust the survival of cancer cells. In addition, miRNA-130a is also differentially expressed in diseases, including lung (15), prostate (16), breast (17), gallbladder (18) and cervical cancers (19).

Currently, there are few studies on the expression of miRNA-130a in glioma, and through RT-qPCR, MTT assay, Transwell migration assay, scratch assay and flow cytometry (FCM), this study tried to prove the expression of miRNA-130a in glioma cells and other biological functions. First of all, RT-qPCR was used to detect the expression of miRNA-130a in the bare, miRNA-130amimics, miRNA-130a-inhibitor and miRNA-scramble groups, and the results showed that after transfection, the expression quantity in the miRNA-130a-mimics group was significantly increased $(\mathrm{P}<0.05)$, indicating that miRNA$130 \mathrm{a}$ is highly expressed in glioma cells. The expression in the miRNA-130a-inhibitor group was obviously decreased, compared with the bare and miRNA-scramble groups $(\mathrm{P}<0.05)$. It is speculated that the functions of glioma cells including proliferation, invasion, migration and apoptosis can be inhibited by inhibiting and decreasing the expression of miRNA-130a in cells. To verify such a speculation, MTT, Transwell migration and scratch assays were conducted. In the cell proliferation assay, from the 2 nd day, the cell proliferation began to be inhibited in the miRNA-130a-inhibitor group, and as of the 7th day, it showed significant differences compared to those in the other 3 groups. This fact, reveals that the inhibition of miRNA-130a can effectively reduce the proliferation of cancer cells. Wang et al (20) reported that miRNA-130a is highly expressed in non-small cell lung cancer and antagonizes Gax gene which plays an inhibiting role on proliferation, migration and angiogenesis of the endothelial cells. This shows that the expression of the same miRNA is identical in different cancers. Then, Transwell migration and scratch assays further confirmed this view. According to the results, the invasion and migration abilities in the miRNA-130a-mimics group were significantly improved, compared to those in the other 3 groups $(\mathrm{P}<0.05)$. Among U-87MG cells, the proportions of apoptotic cells in the blank and negative control groups were 6.88 and $7.54 \%$, respectively. After the transfection of miRNA-130a, they were increased to 31.84 and $30.31 \%$, respectively. The experiment proved that the expression of miRNA-130a can weaken the abilities of cell proliferation and apoptosis. Apoptosis plays a critical role in the occurrence and development of tumors and is an important method on tumor treatment.

In conclusion, this study verified that miRNA-130a is highly expressed in glioma through the experiments. Moreover, the downregulated miRNA-130a inhibits the proliferation of glioma cell lines and promotes apoptosis. This indicates that miRNA-130a can be considered a candidate target for the gene therapy of glioma.

\section{Acknowledgements}

Not applicable.

\section{Funding}

No funding was received.

\section{Availability of data and materials}

The datasets used and/or analyzed during the present study are available from the corresponding author on reasonable request.

\section{Authors' contributions}

KL and HS conceived and designed the study. KL, SZ and $\mathrm{SB}$ were responsible for the collection and analysis of the the in vitro data. KL, HS and SW interpreted the data and drafted the manuscript. HS and SZ revised the manuscript critically for important intellectual content. All authors read and approved the final study. 


\section{Ethics approval and consent to participate}

The study was approved by the Ethics Committee of The Second Nanning People's Hospital (Nanning, China).

\section{Patient consent for publication}

Not applicable.

\section{Competing interests}

The authors declare that they have no competing interests.

\section{References}

1. Sathornsumetee S, Reardon DA, Desjardins A, Quinn JA, Vredenburgh JJ and Rich JN: Molecularly targeted therapy for malignant glioma. Cancer 110: 13-24, 2007.

2. Clarke J, Butowski N and Chang S: Recent advances in therapy for glioblastoma. Arch Neurol 67: 279-283, 2010.

3. Valle-Folgueral JM, Mascarenhas L, Costa JA, Vieira F, Soares-Fernandes J, Beleza P and Alegria C: Giant cell glioblastoma: Review of the literature and illustrated case. Neurocirugia (Astur) 19: 343-349, 2008.

4. Iorio MV and Croce CM: MicroRNA dysregulation in cancer: Diagnostics, monitoring and therapeutics. A comprehensive review. EMBO Mol Med 4: 143-159, 2012.

5. Prosdocimo G and Giacca M: Manipulating the proliferative potential of cardiomyocytes by gene transfer. In: Adult Stem Cells. Di Nardo P, Dhingra S and Singla D (eds). Vol 1553 Humana Press, New York, NY, pp41-53, 2017. https://doi. org/10.1007/978-1-4939-6756-8_4.

6. Qiu S, Lin S, Hu D, Feng Y, Tan Y and Peng Y: Interactions of miR-323/miR-326/miR-329 and miR-130a/miR-155/miR-210 as prognostic indicators for clinical outcome of glioblastoma patients. J Transl Med 11: 10, 2013.

7. Allen M, Bjerke M, Edlund $\mathrm{H}$, Nelander S and Westermark B: Origin of the U87MG glioma cell line: Good news and bad news. Sci Transl Med 8: 354re3, 2016.

8. Yabroff KR, Harlan L, Zeruto C, Abrams J and Mann B: Patterns of care and survival for patients with glioblastoma multiforme diagnosed during 2006. Neuro-oncol 14: 351-359, 2012.

9. Ohgaki H, Dessen P, Jourde B, Horstmann S, Nishikawa T, Di Patre PL, Burkhard C, Schüler D, Probst-Hensch NM, Maiorka PC, et al: Genetic pathways to glioblastoma: A population-based study. Cancer Res 64: 6892-6899, 2004.
10. Streiff MB, Ye X, Kickler TS, Desideri S, Jani J, Fisher J and Grossman SA: A prospective multicenter study of venous thromboembolism in patients with newly-diagnosed high-grade glioma: Hazard rate and risk factors. J Neurooncol 124: 299-305, 2015.

11. Zhang Y, Yang P and Wang XF: Microenvironmental regulation of cancer metastasis by miRNAs. Trends Cell Biol 24: 153-160, 2014.

12. Fujita Y, Yoshioka $Y$ and Ochiya T: Extracellular vesicle transfer of cancer pathogenic components. Cancer Sci 107: 385-390, 2016.

13. Li BS, Zuo QF, Zhao YL, Xiao B, Zhuang Y, Mao XH, Wu C, Yang SM, Zeng H, Zou QM, et al: MicroRNA-25 promotes gastric cancer migration, invasion and proliferation by directly targeting transducer of ERBB2, 1 and correlates with poor survival. Oncogene 34: 2556-2565, 2015.

14. Kovaleva V, Mora R, Park YJ, Plass C, Chiramel AI, Bartenschlager R, Döhner H, Stilgenbauer S, Pscherer A, Lichter P, et al: miRNA-130a targets ATG2B and DICER1 to inhibit autophagy and trigger killing of chronic lymphocytic leukemia cells. Cancer Res 72: 1763-1772, 2012.

15. Liu L, Nie J, Chen L, Dong G, Du X, Wu X, Tang Y and Han W: The oncogenic role of microRNA-130a/301a/454 in human colorectal cancer via targeting Smad4 expression. PLoS One 8: e55532, 2013

16. Fujita Y, Kojima T, Kawakami K, Mizutani K, Kato T, Deguchi T and Ito M: miR-130a activates apoptotic signaling through activation of caspase- 8 in taxane-resistant prostate cancer cells. Prostate 75: 1568-1578, 2015

17. Pan Y, Wang R, Zhang F, Chen Y, Lv Q, Long G and Yang K: MicroRNA-130a inhibits cell proliferation, invasion and migration in human breast cancer by targeting the RAB5A. Int J Clin Exp Pathol 8: 384-393, 2015.

18. Ma MZ, Li CX, Zhang Y, Weng MZ, Zhang MD, Qin YY, Gong W and Quan ZW: Long non-coding RNA HOTAIR, a c-Myc activated driver of malignancy, negatively regulates miRNA-130a in gallbladder cancer. Mol Cancer 13: 156, 2014.

19. He L, Wang HY, Zhang L, Huang L, Li JD, Xiong Y, Zhang MY, Jia WH, Yun JP, Luo RZ, et al: Prognostic significance of low DICER expression regulated by miR-130a in cervical cancer. Cell Death Dis 5: e1205, 2014.

20. Wang XC, Tian LL, Wu HL, Jiang XY, Du LQ, Zhang H, Wang YY, Wu HY, Li DG, She Y, et al: Expression of miRNA-130a in nonsmall cell lung cancer. Am J Med Sci 340: 385-388, 2010. International (CC BY-NC-ND 4.0) License. 